\title{
La santé est au cour de la politique et la politique au cœur de la santé
}

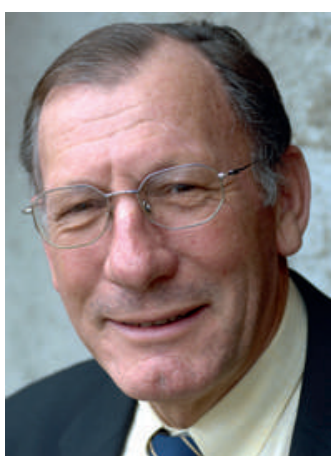

Jean Martin
Les soins de santé sont au cœur de la politique. C'est particulièrement manifeste en ce printemps 2012. Chez nous, la disposition légale sur les soins intégrés/ managed care a mis en évidence des options, des craintes et des démarches divergentes au sein du corps médical. Le projet de loi fédérale sur la prévention a vécu des péripéties étonnantes, avec le refus d'entrée en matière du Conseil des Etats à fin 2011 et la reprise en ce moment des travaux des Chambres. Faut-il ici rappeler combien le contexte de vie dans ses diverses dimensions, socio-économique, culturelle, physicochimique (pollutions) influence la santé et demande des mesures préventives adéquates? Mais le fait est que les sociétés libérales au sens large, comme la nôtre, y sont a priori peu favorables. Elles préfèrent attendre et voir, rechignent aux «gardefous» nécessaires au nom de motifs souvent caricaturaux invoquant la liberté individuelle. Ceci au risque de dégâts ultérieurs dont il faudra payer les conséquences, mais sans pouvoir réparer vraiment les dommages humains voire sociétaux. Judicieusement, la FMH soutient la loi sur la prévention et on veut croire que, après tant de débats, elle sera adoptée.

Aux Etats-Unis, des allégations similaires de limites à la liberté de chacun sont invoquées pour s'opposer à l' «Affordable Care Act» («Obamacare» pour ses détracteurs). La loi a été promulguée il y a deux ans mais certains de ses points viennent d'être examinés lors d'auditions de la Cour suprême. Il s'agit de savoir si le universal individual mandate qu'elle prévoit est conforme à la Constitution. La disposition contestée est celle qui prévoit que chaque Américain a le devoir, d'une manière ou de l'autre, de s'assurer contre la maladie. On voit à ce propos des argumentations qui mettent sur le même plan, en les opposant, la liberté d'acheter une voiture ou de prendre comme on l'entend des loisirs, et l'instauration d'une garantie pour tous de soins minimalement adéquats. L'accès aux soins de qualité n'étant pas considéré comme un droit social majeur, comme en général en Europe, mais comme une commodity qu'on se paie si on en a les moyens et dont on se passe sinon. Le caractère fondamentalement politique du débat est illustré par le fait que les arrêts de tribunaux inférieurs sur le sujet sont tombés strictement selon l'appartenance partisane des juges. Comme la Cour suprême compte cinq républicains et quatre démocrates, il y a un vrai risque que ce qui est décrit comme la législation la plus importante en matière sociale dans le pays depuis quarante ans soit invalidée pour un motif qui, de ce côté-ci de l'Atlantique, paraît inepte.

$\mathrm{Au}$ plan international: surprise tout récemment que la désignation par les Etats-Unis d'un médecin comme candidat à la présidence de la Banque mondiale, un des organismes principaux censés contribuer au développement dans les pays pauvres (il peut y avoir d'autres candidats mais c'est la pratique jusqu'ici de la communauté internationale de nommer le candidat US). Il s'agit du Prof. Jim Yong Kim, 53 ans, Américain d'origine coréenne, médecin de santé publique et président d'université. Après avoir co-fondé l'ONG «Partners in Health» qui a mis en place dans plusieurs pays des programmes efficaces et économiques de lutte contre les grandes maladies infectieuses, il a travaillé pour l'OMS de 2003 à 2006 tout en enseignant à Harvard. Alors que la présidence (très politique) de la Banque mondiale était généralement réservée à une personne issue de la finance, on peut espérer que cette institution montrera plus d'intérêt que par le passé, et un intérêt plus compétent, à la santé comme facteur primordial du développement. Le Dr Jim Yong Kim aura probablement à l'esprit cette phrase de Martin Luther King: «De toutes les formes d'inégalité, l'injustice en matière de soins de santé est la plus inhumaine.»

La politique a toujours une dimension éthique ou en tout cas devrait en avoir une. Je mentionne pour terminer un ouvrage sur la bioéthique dans le monde [1]. Il étudie comment, dans divers continents et régions, on n'accorde pas le même poids à tel ou tel des principes éthiques majeurs. En particulier en ce qui concerne une sorte d'absolutisme de l'autonomie individuelle. En Afrique et en Asie, le groupe (la famille élargie voire la communauté) joue dans les décisions un rôle que nos conceptions occidentales n'acceptent pas aisément [2]. Cela étant, on doit bien sûr refuser qu'une personne soit soumise indûment aux décisions d'autres; mais une sensibilité à des cadres de référence différents est indispensable si on entend élaborer des modèles répondant aux besoins et aux circonstances de vie de tous.

Dans un autre livre récent traitant d'éthique [3], C. Pelluchon affirme que, dans les circonstances actuelles de notre monde, la responsabilité personnelle de chacun doit prévaloir sur sa seule liberté. Y réfléchir!

Jean Martin, membre de la rédaction et de la Commission nationale d'éthique 